\title{
The shRNA-mediated silencing of $V E G F-C$ illustrates its role in proliferation, chemosensitization, tumor colonization, and anchorage independence
}

\author{
Poonam Tambe $^{1 \dagger}$, Indira Purohit ${ }^{1 \dagger}$, Daxa Suneja ${ }^{2}$, \\ Shraddha More ${ }^{2}$, Priti Desai ${ }^{1}$, Neeta Shrivastava ${ }^{1,2 *}$ \\ ${ }^{1}$ B.V. Patel Pharmaceutical Education Research and Development (PERD) Centre, Ahmedabad, Gujarat, India \\ ${ }^{2}$ National Institute of Pharmaceutical Education and Research (NIPER), \\ B.V. Patel Pharmaceutical Education Research and Development (PERD) Centre, Ahmedabad, Gujarat, India \\ ${ }^{\dagger}$ Both authors had contributed equally; ${ }^{\ddagger}$ Registered Ph.D. student of Nirma University
}

\begin{abstract}
Vascular endothelial growth factor C (VEGF-C) is known to stimulate growth of endothelial cells to form tumor-associated lymphatics, which leads to the migration of tumor cells to distant places. However, the function of VEGF-C is not limited to lymph angiogenesis only. VEGF-C is produced by breast cancer cells and binds to the receptors present on them and to lymphatic endothelial cells. The binding of VEGF-C to its receptors on a cancer cell is referred to as autocrine signaling. This ligand-receptor binding, activates a cascade of events that are different from angiogenesis. Moreover, autocrine transmission of signals may facilitate the growth, migration, and drug sensitivity of cancer cells, including the malignancy of the breast. In this study, we investigated the effect of inhibition of $V E G F-C$ gene expression via RNAi. A vector-based siRNA was constructed and transfected into breast cancer cell line MDA-MB-231. VEGF-C gene expression was analyzed via RT-PCR. In vitro proliferation, soft agar colony formation, and scratch healing assays were performed. A relative decrease in $V E G F-C$ gene expression observed in transfected cells indicated the silencing effect of shRNA. Due to unavailability of VEGF-C protein, the replicative potential of cancer cells decreased. No colonies on soft agar formed by these tumor cells were observed, which explains their incapability of being anchorage independent. Similarly, scratch healing and 3-(4, 5- dimethylthiazol-2-yl)-2, 5-diphenyltetrazolium bromide (MTT) assay showed a non-migratory and drug-sensitive nature of the transfected cells. All our results indicate that abundance of VEGF-C in tumor microenvironment will affect various non-angiogenic cell functions such as proliferation, migration, colonization, and chemoresistance.
\end{abstract}

Key words: VEGF-C, breast cancer, lymphangiogenesis, metastasis, shRNA, RNAi

\section{Introduction}

Metastasis to regional lymph nodes and distant organs such as bones or lungs associated with breast cancer is a major cause of mortality (Eccles et al., 2007). The factors contributing to metastasis are molecular aberrations occurring within both the tumor cells and the tumor microenvironment. In short, the process comprises detachment of tumor cells from their primary site of location, invasion into the tumor stroma, intravasation into adjacent blood vessels or lymphatics, survival in the bloodstream, extravasation into and colonization of the target organ, and, finally, metastatic outgrowth (Gupta and Massagué, 2006). Certain studies have suggested the presence of intra-tumoral and peritumoral lymphatics supporting the findings that the development of lymphatics is restored during carcinogenesis (Chaffer and Weinberg, 2011) which is crucial in systemic dissemination (Nguyen et al., 2009). Various studies highlighted the role of vascular endothelial growth factor C (VEGF-C) as a major prolymphangiogenic player ( $\mathrm{Su}$ et al., 2006). The binding of VEGF-C and vascular endothelial growth factor receptor-3 (VEGF-CR-3) stimulates proliferation and migration of lymphatic endothelial cells and, in turn, augments the assembly of new lymphatic vessels. The

\footnotetext{
* Corresponding author: B.V. Patel Pharmaceutical Education Research and Development Centre, SG Highway, Thaltej, Ahmedabad (380054), Gujarat, India; e-mail: neetashrivastava_perd@yahoo.co.in
} 
activation of VEGF-CR-3 not only stimulates proliferation of tumor cells but also inhibits apoptosis and decreases the sensitivity to chemotherapeutic drug by increasing the Bcl-2/ Bax ratio (Nguyen et al., 2009; Mohammed et al., 2009). This can be attributed to the expression and activation of other receptors such as VEGF-CR-2, Neuropilin-1 and 2 on tumor cells (Timoshenko et al., 2007). These observations suggest that in addition to the major lymphangiogenic function, VEGF-C may contribute as an autocrine molecule directly affecting the functions of certain cancer cells exposing any of these VEGF-C binding receptors on their surfaces. The results obtained in these studies suggest some non-lymphangiogenic functions of VEGF-C which consequently result in tumor cell self-sufficiency. The treatments aimed specifically to block tumor dissemination through the lymphatic network could, in principle, provide an independent therapy for some cancers, or at least be an adjunct treatment to existing chemotherapy (Sun et al., 2008). In this study, we suggest that the inhibition of $V E G F-C$ can improve its utility as a therapeutic target. Due to the fact that very little information was available on autocrine functions of VEGF-C on breast cancer cells, this study was designed to enumerate different accessory functions of VEGF-C.

\section{Materials and methods}

\section{Design of SiRNA targeting VEGF-C gene sequence}

DNA databases at Ensembl (release 54) were used to search the genomic regions, transcripts, and products in the complete coding sequence (cds) of VEGF-C gene. Its c-DNA sequence was used as a query in siRNA TargetFinder tool (Ambion, USA). The specific shRNA was taken according to the published guidelines (Ui-Tei et al., 2004). The general guidelines as enumerated by an online tool were as follows: ending of siRNA sequence with TT or UU, maximum GC content allowed to be $50 \%$, and 4 or more C's or A's in a row to be avoided. The more prevalent siRNAs were selected on the basis of guidelines such as \%GC content (47-49\%). These were run for BLAST analysis to avoid off targeting (Jackson et al., 2006). The candidate siRNA targeting at position 381 in the mRNA of $V E G F-C$ gene with $47 \%$ GC content [GenBank. NM005429] was selected. For the synthesis of siRNA in the cells, the selected candidate siRNA sequences were converted into complementary DNA sequences. These sequences were preceded by Pol III-type pro- moter sequence of $\mathrm{H} 1$ as an expression cassette. These sequences were custom synthesized as an insert vector [Supplementary Figure 1] (Geneart, Germany).

siRNA sequence:

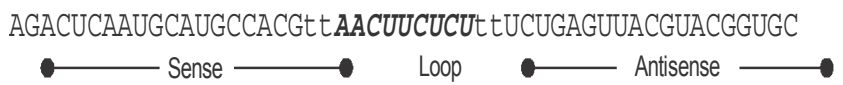

\section{Construction of small interfering $R \boldsymbol{R A}$ expression vectors}

For the purpose of the study, a long-term inhibition of target mRNA was obtained. Briefly, the selected sequence was cloned into a shRNA expression vector, pSUPER.neo (backbone vector) (McManus et al., 2002). Because the backbone vector harbored U6-driven expression of siRNA gene, the siRNA sequence was cloned using Eco RI and Hind III sites into the backbone vector; thus, the expression occurred under the control of $\mathrm{H} 1$ promoter present in the cassette (Luo et al., 2007). The final vector (VEGF C/shRNA, psNIPERD_H1C1) [Supplementary Figure 2] was isolated with GenElute plasmid isolation kit (Sigma Aldrich), confirmed by a restriction analysis and sequencing.

\section{Cell culture and transfection of in vitro mammary tumor cells}

VEGF-C gene overexpression and highly metastatic cell line of breast cancer were used. Human breast cancer cell line MDA-MB-231 (Source: National Centre for Cell Science, Pune, India). The cells were cultured in RPMI-1640 (Gibco) media supplemented with $10 \%$ fetal bovine serum (FBS, Gibco), penicillin $\left(10^{7} \mathrm{U} / \mathrm{l}\right)$, and streptomycin $(10 \mathrm{mg} / \mathrm{l})$. Transfections were performed at $70 \%$ confluency in 6 -well plates (Corning). Total of $2 \times 10^{6}$ cells $/ 200 \mu 1$ MDA-MB-231 mammary tumor cells were transfected with $5 \mu \mathrm{g}$ DNA (psNIPERDH1C1) using electroporation (Gene pulser, BioRad) with a single pulse of $75 \mathrm{~V}$ for $30 \mathrm{~ms}$. The transfected cells were selected with $1.2 \mathrm{mg} / \mathrm{ml} \mathrm{G} 418$-sulfate $48 \mathrm{~h}$ post-transfection. In each experiment, the vector (pSUPER.neo) and mock electroporated cells were included as controls. Stably transfected MDA-MB-231-siVEGF-C, MDA-MB-231-p SUPER.neo, and mock cells were obtained and routinely maintained in the selection media.

\section{Messenger RNA analysis}

The total cellular RNA was isolated from MDA-MB231-si VEGF-C and MDA-MB-231-pSUPER.neo and mock 
cells using Trizol reagent (Invitrogen), and cDNA was subsequently synthesized using $5 \mu \mathrm{g}$ of total RNA, random hexamer primers, and Revert Aid H minus Reverse Transcriptase (Fermentas). Using gene-specific primers, PCR was done for $V E G F-C$ gene forward primer $5^{\prime}$-CATGTACGAACCGCCAGAA-3', reverse primer 5'-TTGTTAGCATGGACCCACAA-3' with amplicon size of 212 bp. Beta actin was used as an internal standard and amplified with $5^{\prime}$-TGTGATGGTGGGAATGGGTCAG-3' 5' $^{\prime}$-TTTGATGTCACGCACGATTTCC-3' primer pair, and a predicted product of $512 \mathrm{bp}$ (Kramer and Coen, 2001).

\section{In vitro proliferation assay}

In order to measure the inhibition of cell proliferation by VEGF-C shRNA, 1,000 cells were seeded into 96well flat-bottomed plates in triplicates. Proliferative activity was determined at regular intervals of $72,48,24$, 12 , and $4 \mathrm{~h}$ by the 3-(4,5-dimethylthiazol-2-yl)-2,5-diphenyltetrazolium bromide (MTT) assay using a microtiter plate reader (Bio-Tek Instruments, Inc., Winooski, VT) at $570 \mathrm{~nm}$ (Niles et al., 2008). The growth stimulation or inhibition was calculated as $(\%)=\left[(\mathrm{A} / \mathrm{B})^{*} 1^{*} 100\right]$, where $A$ is the absorbance of treated cells and $B$ is the absorbance of untreated control cells (Schröterová et al., 2009). Each sample was run in triplicates and was repeated thrice.

\section{Scratch wound assay}

Tumor cells migrate to distant places under the effect of VEGF-C signal transduction. The inhibition of migration was checked by conventional in vitro scratch wound assay. Essentially, in this method, the cells from a monolayer are scrapped with the help of a sterile tip so that a gap is created. The cells at the boundary of the gap start migrating till the gap is resealed. Images of test and controls are captured at regular intervals and compared to check the rate of migration. The percentage of wound area remaining at each time point was calculated using Image J software (Liang et al., 2007). Each sample was run in triplicates and was repeated thrice.

\section{Soft agar assay for colony formation}

The cells that acquire a migratory phenotype are able to form colonies at different sites (metastatic foci). This can be assessed in vitro by colony-forming assay. A base layer of $0.5 \%$ agar, 1X RPMI 1640 with 10\% FBS (1 ml) was prepared in a 6 -well plate. About $0.5 \times 10^{4}$ transfected and mock cells were resuspended in $1 \mathrm{ml}$ of $0.35 \%$ agarose, 1X RPMI, 10\% FBS and poured as a top layer above the base layer. An additional $1 \mathrm{ml}$ of RPMI 1640 with 10\% FBS was added to each well and the plates were incubated at $37^{\circ} \mathrm{C}, 5 \% \mathrm{CO}_{2}$. Images of colonies were captured under an inverted microscope at $100 \times$ total magnification (Anderson et al., 2007). Images of colonies in soft agar were counted and the mean colony area was calculated using web-based Wimasis software. Each sample was run in triplicates and was repeated thrice.

\section{Metastatic foci formation assay}

The cells that acquire a migratory phenotype are able to form colonies at different sites (metastatic foci). This is assessed in vitro by focus formation assay. This assay is based on the ability of a single cell to form individual colonies. It was expected that after the treatment with VEGF-C shRNA, the cells would lose their unlimited divisional capacity. To test these single cell suspensions at very high cell dilutions (up to $5000 \times$ ), the cells were placed into a 6 -well plate and incubated for 7 days. Following the incubation, they were stained with Giemsa, and the images were captured (Franken et al., 2006). Images were analyzed using ImageJ software for quantitative data analysis. Each cell sample was run in triplicates and was repeated thrice.

\section{Chemosensitivity assay}

shRNA-mediated inhibition of $V E G F-C$ can also increase the sensitivity to chemotherapeutic drugs. So to check whether VEGF-C sequence can mimic the same phenomenon, $1 \times 10^{4}$ cells were placed onto 96 wells plate in triplicates (Mock, pSUPER.neo- and psNIPERDH1C1transfected cells). The cells were treated with doxorubicin for $24 \mathrm{~h}$. MTT $(0.5 \mathrm{mg} / \mathrm{ml}$ in phenol-red free medium) ( $100 \mu \mathrm{l} /$ well $)$ was added and further incubated for $3 \mathrm{~h}$ at $37^{\circ} \mathrm{C}$ and then MTT was removed from the wells. To solubilize formazan crystals in the wells, DMSO (100 $\mu 1 /$ well) was added and mixed gently. Absorbance was determined at $570 \mathrm{~nm}$ in a microplate reader (Biotek) and expressed as \% Cell viability (Burton, 2005). It was repeated thrice. The results were expressed as a mean \pm standard error of mean (SEM).

\section{Statistical analysis}

A statistical analysis was performed using SPSS software (Release 11.0, SPSS Inc.) and GraphPad Prism 


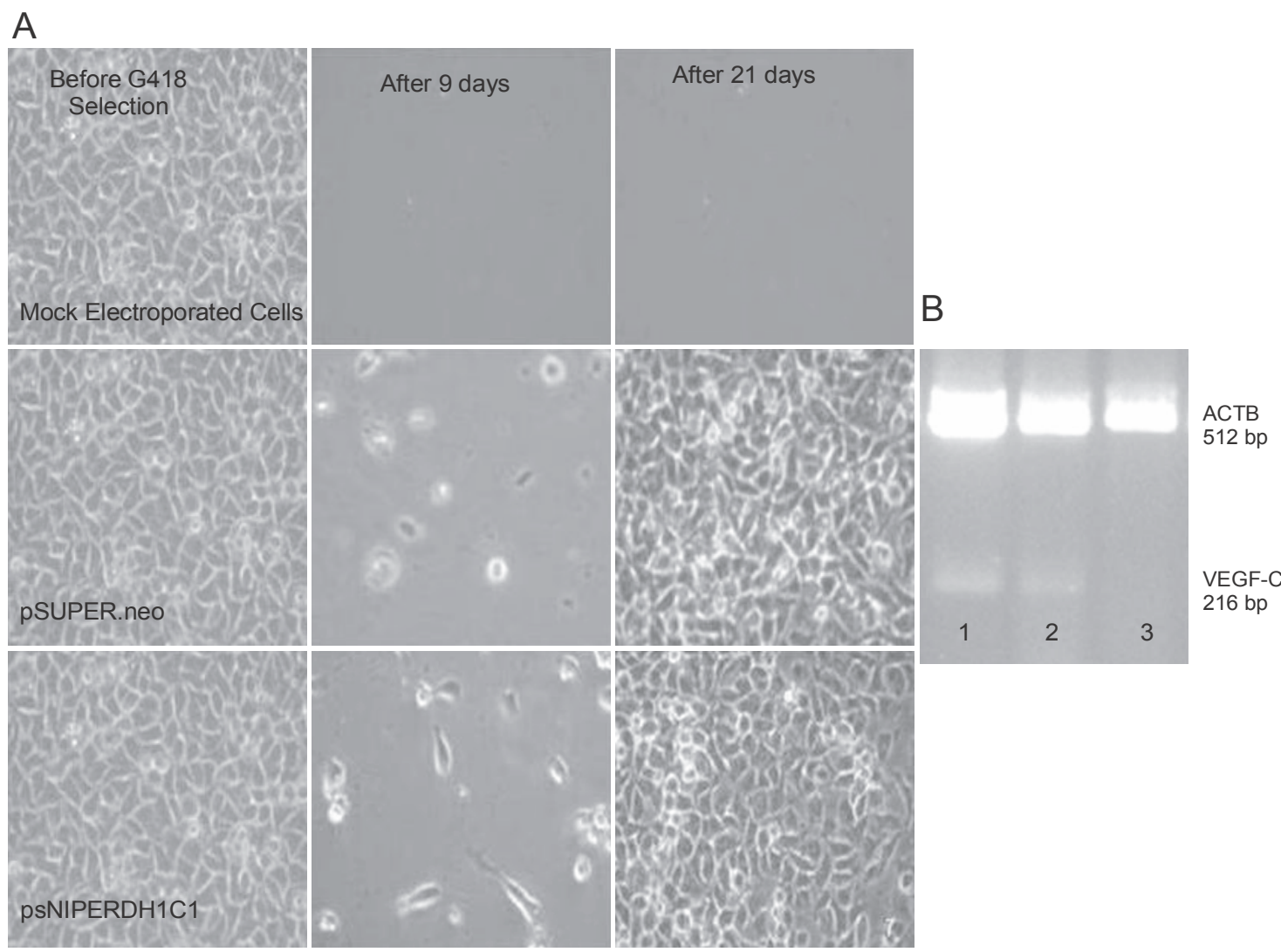

Fig. 1. Transfection and RT_PCR.A) Representative fields of cells before and B) after the selection on G418 containing medium up to 21 days: 1) PCR products from RNA of Mock control, 2) pSUPER.neo-transfected cells, 3) psNIPERDH1C1cells. Lanes 1 and 2 both showed the presence of VEGF-C and $\beta$ actin, while psNIPERDH1C1-transfected cells (lane 3 ) showed only $\beta$ actin band. $p>0.05$ for $\beta$ actin in all the three samples whereas $p<0.05$ for $V E G F-C$ in control versus psNIPERDH1C1

(version 6). The data were expressed as the mean \pm SEM or SD as specified. $p$ values were determined by an appropriate ANOVA and Dunnett's multiple comparison tests. Significant codes are as follows: “*” if $p<0.05$, “**” if $p<0.01$, “***” if $p<0.001$, and “****” if $p<0.0001$.

\section{Results}

\section{Construct screening and transfection}

The recombinant plasmid (psNIPERDH1C1) was confirmed by a restriction analysis (Eco RI/Hind III). The digested fragments (4472 bp and $170 \mathrm{bp}$ ) were separated on agarose gels [ $1.5 \%$ in $0.5 \times \mathrm{TAE}]$. The transfected cells were observed under a phase contrast microscope (Fig. 1A).

\section{VEGF-C $m R N A$ analysis}

Dunnett's multiple comparison test of semi-quantitative gel densitometry of RT-PCR gel showed a significant decrease in $V E G F-C$ mRNA levels in $V E G F-C /$ shRNA transfected cells $(p<0.05)$ while steady state levels of the housekeeping gene $\beta$ actin remained relatively unchanged $(p>0.05)$ (Fig. 1B).

\section{Effect of VEGF-C/shRNA on proliferation and chemo- sensitivity of human mammary adenocarcinoma cells}

VEGF-C implies an autocrine and paracrine growthpromoting effects, and hence it may be released into the growth medium, thereby inducing migration and proliferation. Consequently, we hypothesized that VEGF-C shRNA may reduce migration and proliferative potential of MDA-MB-231 cells. Cancer cells acquire very high proliferation ability which is attributed to overexpression of VEGF-C gene. As a result, VEGF-C gene silencing should decrease their proliferation which is reflected in reduced viable cell population during MTT assay. psNIPERDH1C1-transfected cells showed a significant decrease in the OD at different time intervals as compared to mock and pSUPER.neo-transfected cells which in 


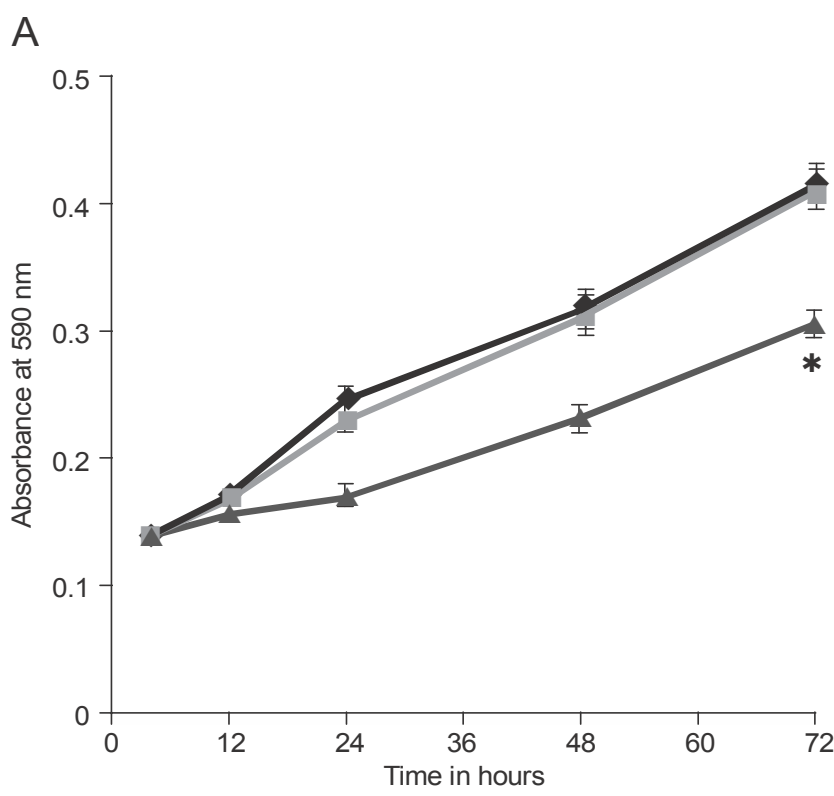

B

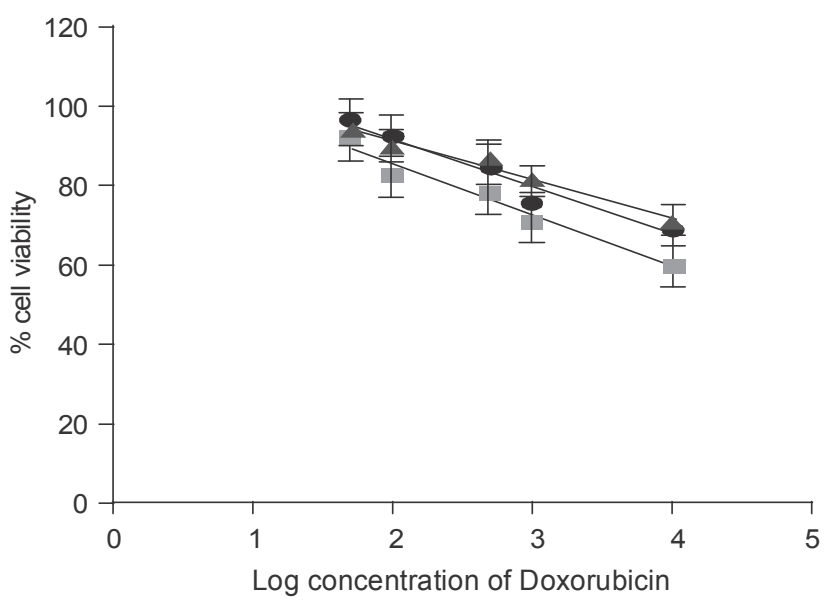

Fig. 2. Effect of silencing on cell proliferation and chemosensitivity; A) MTT assay with mock (black diamond), pSUPER.neotransfected (gray square) cells and psNIPERDH1C1-transfected cells (gray triangles) $\left(p^{*}<0.05\right.$, bars s.d); B) Doxorubicin sensitivity of psNIPERDH1C1-transfected cells (gray square, $y=$ $-13.03 \mathrm{x}+111.6, \mathrm{R} 2=0.964)$, mock (dark circles, $y=-12.17 \mathrm{x}$ $+116.5, \mathrm{R} 2=0.951)$ cells, and pSUPER.neo-transfected cells (gray triangles, $y=-9.660 \mathrm{x}+110.7, \mathrm{R} 2=0.968)\left(p^{*}<0.05\right.$, bars s.e.m)

dicated reduced tumor cell proliferation due to $V E G F-C$ gene silencing (Fig. 2A). psNIPERDH1C1-mediated inhibition of VEGF-C gene expression increased doxorubicin sensitivity in MDA MB 231. The increment in sensitivity reduced the cell viability whereas the mock transfected and vector control had more population of viable cells. This shows that chemoresistance can be reversed or reduced by $V E G F-C$ gene silencing (Fig. 2B).
VEGF-C gene silencing decreases migration of breast cancer cells

The scratch and wound assay displayed a migratory nature of the mock and pSUPER.neo-transfected cells as compared to psNIPERDH1C1-transfected cells after $72 \mathrm{~h}$. The mock and pSUPER.neo-transfected cells migrated toward the site of wound leading to its closure while the gap remained unsealed in case of VEGF-C-silenced cells, as shown in images (Fig. 3A). The quantitative values of wound size were determined by ImageJ software. For multiple comparisons, Dunnett's test was applied. According to this, insignificant difference was observed between mock cells and pSUPER.neo-transfected cells at any time point. Whereas significant difference was observed between gap closure of pSUPER.neo-transfected cells and psNIPERDH1C1-transfected cells (Fig. 3B).

\section{VEGF-C gene silencing inhibits anchorage-independent growth of cells in soft agar assay}

Metastatic cancer cells (circulating in vasculature) are capable of evading attachment-regulated apoptosis, leading to uncontrolled proliferation. The growth of cells in soft agar indicates survival and proliferation of cells in the circulating state, which is a hallmark of malignant cells. The knockdown of VEGF-C gene expression in psNIPERDH1C1-transfected cells reduced the number of colonies, in comparison to mock and pSUPER.neotransfected cells, when cultured in a soft agar medium up to 15 days. The mean number of colonies of psNIPERDH1C1-transfected cells was significantly lesser than these in pSUPER.neo-transfected cells (Fig. $4 \mathrm{~A})$. The difference in the mean area of colonies was significant as calculated by one-way ANOVA (Fig. 4B), while the difference was insignificant when mock and pSUPER.neo-transfected cells were compared by Dunnett's multiple comparison test.

\section{VEGF-C gene silencing decreases foci formation}

The effect of VEGF-C gene silencing on the focusformation ability of mock and psNIPERDH1C1-transfected cells was evaluated. The mock and pSUPER.neotransfected cells showed the maximum number of foci as compared to psNIPERDH1C1-transfected cells (Fig. 4C). The mean number of foci was significantly lesser in $V E G F-C$-silenced cells (Fig. 4D). 

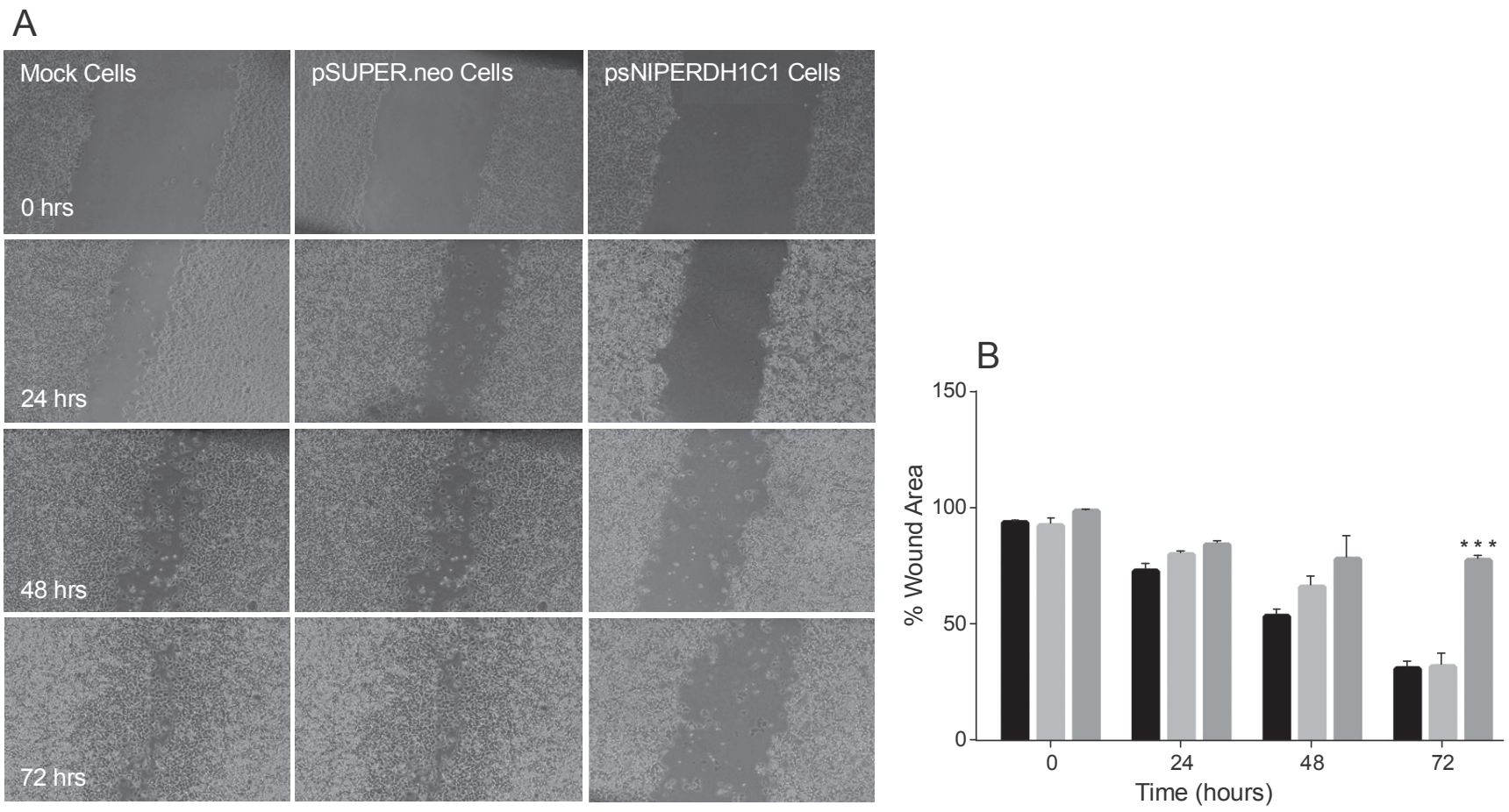

Fig. 3. Scratch wound assay. A) Images from three independent experiments captured at 24,48 , and $72 \mathrm{~h}(40 \times$ total magnification). B) Quantitative values of a wound area left after the migration of cells at different time points. Mock cells (black bars), pSUPER.neo-transfected cells (light gray bars), and psNIPERDH1C1-transfected cells (dark gray bars, bars s.e.m)

\section{Discussion}

The biology of breast carcinoma is highly influenced by the lymphatic system. The lymph nodes are the most common among the preferred sites for tumor dissemination. The process of lymphatic metastasis is a crucial step in the progression of breast cancer, yet the mechanisms that underlie the interactions between tumor cells and lymphatic vessels remain poorly understood (Alitalo and Carmeliet, 2002). The promotion of tumor metastasis via lymphatics has been attributed to excessive VEGF-C signaling through its cognate receptor VEGFCR 3/Flt-4 on lymphatic endothelial cells (Ji, 2006). Lymphatic metastasis also increases resistance to drug treatments (Achen et al., 2006). VEGF-C and Flt-4 axis are synthesized in cancer cell lines, including breast, cervi$\mathrm{cal}$, colorectal, and prostate. The coordinated synthesis of VEGF-C and Flt-4 in some of these tumor cell lines raised the possibility of the existence of an autocrine loop in these cells (Matsuura et al., 2009). Moreover, supplementation of the growth medium with exogenous VEGF-C has shown to increase invasiveness in Kaposi's sarcoma cells that represent VEGF-CR2/3 surface receptors (Su et al., 2008). Favier et al. (2006) have pre- viously demonstrated that VEGF-C can stimulate tumor cell migration by interacting with NRP-2, VEGF-CR 2 and 3. These observations pose VEGF-C as an autocrine molecule which, in addition to the major lymphangiogenic function, may directly affect the functions of certain cancer cells expressing any of these VEGF-C-binding receptors.

In an effort to characterize the effect of VEGF-C on tumor cells, we set out siRNA experiments, using shRNA vector, to repress VEGF-C gene in MDA-MB-231 cell line model of breast cancer. In this way, we were able to successfully reduce the VEGF-C mRNA levels in tumor cells. A comprehensive identification of non-lymphangiogenic functions of VEGF-C may, partly, provide some basis for the application of anti-VEGF-C strategy as a viable therapeutic option, in addition to chemotherapy. Consequently, as a next step, we analyzed the effect of $V E G F-C$ gene silencing on tumor cell proliferation. The findings showed that suppressing the tumor-derived VEGF-C synthesis reduced their own (tumor cells) proliferation thus reducing the number of available tumor cells. The migration is an essential step toward metastasis and invasion. The results indicated that tumor cell 


\section{A}
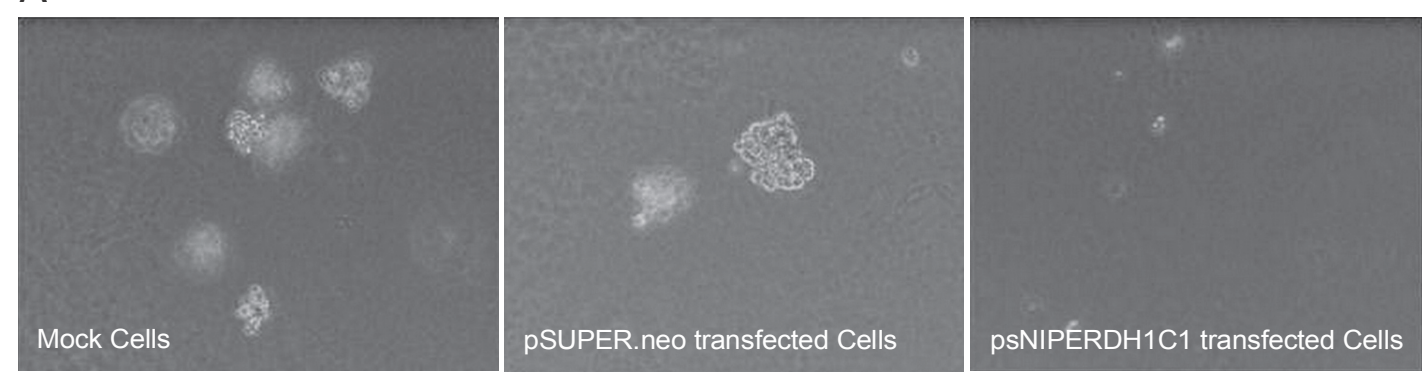

B

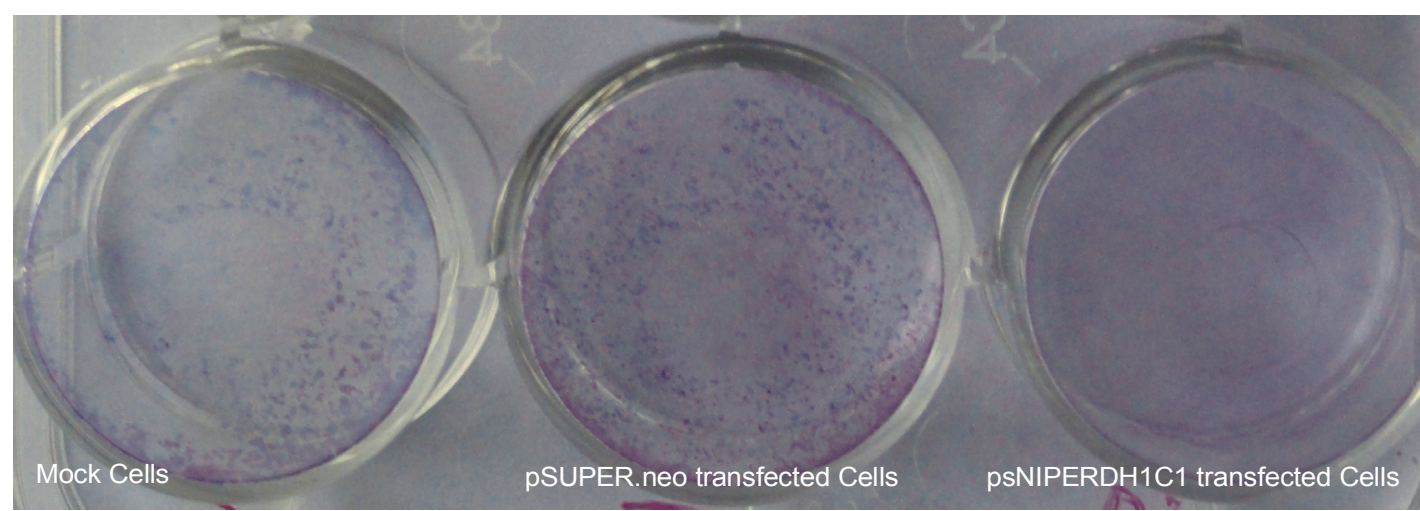

C

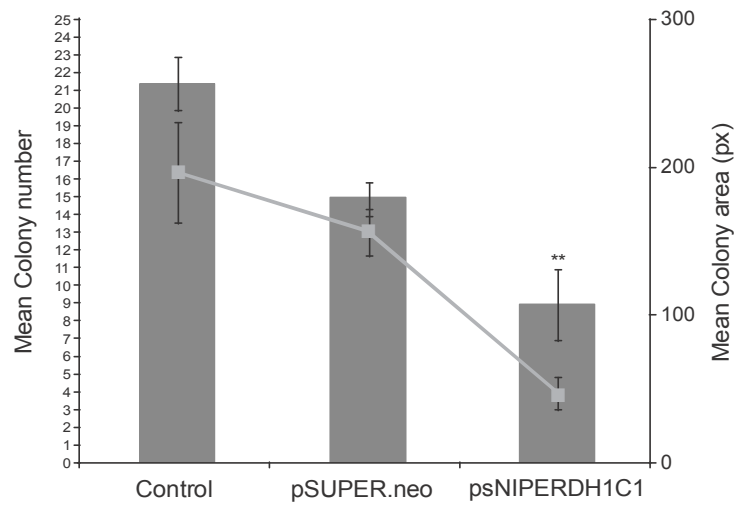

$\mathrm{D}$

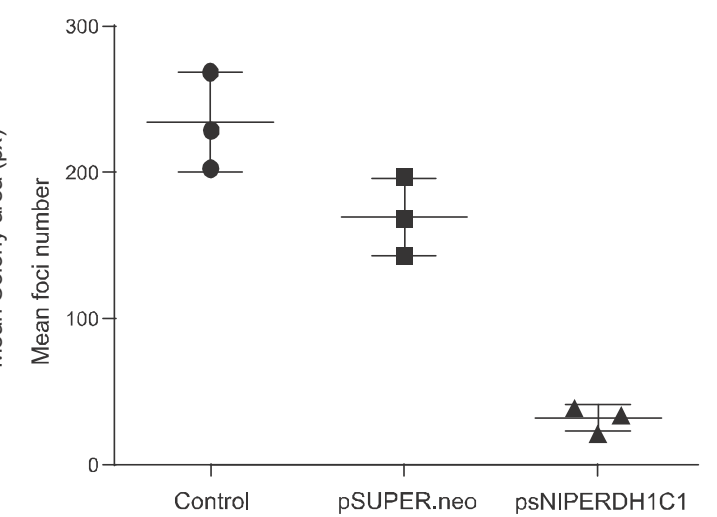

Fig. 4. Colony and Foci formation assays. A) Colonies formed in soft agar by mock, pSUPER.neo-, psNIPERDH1C1-transfected cells at $40 \times$ total magnification. The results are representative of three independent experiments. B) A comparison of the mean colony number (black bars) and the mean colony area in pixels (gray squares). A quantitative analysis of colonies using the WimColony module of Wimas is online software showing a significant decrease in the number of colonies of psNIPERDH1C1transfected cells (bars s.e.m). Mean colony area was also compared between pSUPER.neo- and psNIPERDH1C1-transfected cells, bars s.e.m. C) As compared to Mock and pSUPER.neo-, psNIPERDH1C1-transfected cells formed least foci due to the inhibition of VEGFC gene expression. D) Quantitative analysis of focus-formation assay. Focus-formation images were quantitatively analyzed by ImageJ software. Graphically, there is an insignificant difference which was found between the focusforming capabilities of mock (black circles) and pSUPER.neo-transfected cells (black squares) whereas VEGF-C-silenced cells (black triangles) were significantly different from pSUPER.neo-transfected cells (bars s.e.m.)

migration was negligible in the case of $V E G F-C$ shRNA transfected cells as against the control cells. Migrating cells invade different tissues, demonstrate an anchorageindependent growth, and form new colonies. However, $V E G F-C$-silenced cells showed no colonies at all whereas few colonies were observed in control cells. Anchorage dependency necessitates the availability of base for new translocation. Chemoresistance is also becoming a new clinical problem for breast cancer patients. Recent studies suggest that overexpression of $V E G F-C$ gene leads 
to hyper proliferation and a prolonged survival of leukemias. In addition, low levels of VEGF-C have shown to increase susceptibility toward chemotherapeutics. The purpose of combining conventional cancer therapy with antilymphangiogenic agents is that the antivascular effects of the chemotherapy and radiotherapy are selectively enhanced in the cells of newly formed vessels, for example, when survival signals mediated by VEGF-C are blocked. This study demonstrates a possible use of anti VEGF-C siRNA technology to improve chemotherapy efficacy.

Conclusions: The results of the study support the notion that VEGF-C synthesis by tumor cells not only helps in the development of tumor lymphatics but also indicates non-lymphangiogenic functions of VEGF-C as a result of its autocrine function.

\section{Acknowledgments}

The authors would like to thank Dr. S. Lakshmi and Dr. Reena Agarwal for extending help during this project. The finnancial support from Indian Council of Medical Research (No. 3/1/3/J.R.F.-2008/MPD) is also acknowledged. We also thank Nirma University, Ahmedabad to accept author. Ms Indira Purohit as a $\mathrm{PhD}$ scholar.

\section{References}

Achen M.G., Mann G.B., Stacker S. (2006) Targeting lymphangiogenesis to prevent tumour metastasis. Br. J. Cancer 94: $1355-1360$

Alitalo K., Carmeliet P. (2002) Molecular mechanisms of lymphangiogenesis in health and disease. Cancer Cell 1: 219227.

Anderson S.N., Towne D.L., Burns D.J., Warrior U. (2007) A high-throughput soft agar assay for identification of anticancer compound. J. Biomol. Screen. 12: 938-945.

Burton J.D. (2005) The MTT assay to evaluate chemosensitivity. In: Methods in molecular medicine. Ed. Blumenthal R.D., New Jersey: Humana Press: 69-78.

Chaffer C.L., Weinberg R.A. (2011) A Perspective on Cancer Cell Metastasis. Science 331: 1559-1564.

Eccles S., Paon L., Sleeman J. (2007) Lymphatic metastasis in breast cancer: importance and new insights into cellular and molecular mechanisms. Clin. Exp. Metastasis 24: 619 636.

Favier B., Alam A., Barron P., Bonnin J., Laboudie P., Fons P., Mandron M., Herault J.P., Neufeld G., Savi P. et al. (2006) Neuropilin-2 interacts with VEGFR-2 and VEGFR-3 and promotes human endothelial cell survival and migration. Blood 108: 1243-1250.

Franken N.P., Rodermond H.M., Stap J., Haveman J., van Bree C. (2006) Clonogenic assay of cells in vitro. Nat. Protoc. 1: 2315-2319.

Grimm D. (2009) Small silencing RNAs: State-of-the-art. Adv. Drug Deliv. Rev. 61: 672-703.
Gupta G.P., Massagué J. (2006) Cancer Metastasis: Building a Framework. Cell 127: 679-695.

Hughes B. (2008) Cancer: Tuning anti-angiogenesis. Nat. Rev. Drug Discov. 7: 18-19.

Jackson A.L., Burchard J., Leake D., Reynolds A., Schelter J., Guo J., Johnson J.M., Lim L., Karpilow J., Nichols K. et al. (2006) Position-specific chemical modification of siRNAs reduces "off-target" transcript silencing. RNA 12: 1197-1205.

Ji R.C. (2006) Lymphatic endothelial cells, tumor lymphangiogenesis and metastasis: New insights into intratumoral and peritumoral lymphatics. Cancer Metastasis Rev. 25: 677-694.

Kramer M.F., Coen D.M. (2001) Enzymatic Amplification of DNA by PCR: Standard Procedures and Optimization. In Current Protocols in Molecular Biology. Hoboken, NJ: John Wiley and Sons, Inc.

Liang C.-C., Park A.Y., Guan J.-L. (2007) In vitro scratch assay: a convenient and inexpensive method for analysis of cell migration in vitro. Nat. Protoc. 2: 329-333.

Luo Q., Kang Q., Song W.X., Luu H.H., Luo X., An N., Luo J., Deng Z.L., Jiang W., Yin H. et al. (2007) Selection and validation of optimal siRNA target sites for RNAi-mediated gene silencing. Gene 395: 160-169.

Matsuura M., Onimaru M., Yonemitsu Y., Suzuki H., Nakano T., Ishibashi H., Shirasuna K., Sueishi K. (2009) Autocrine loop between vascular endothelial growth factor (VEGF)-C and VEGF receptor-3 positively regulates tumor-associated lymphangiogenesis in oral squamoid cancer cells. Am. J. Pathol. 175: 1709-1721.

McManus M.T., Petersen C.P., Haines B.B., Chen J., Sharp P.A. (2002) Gene silencing using micro-RNA designed hairpins. RNA 8: 842-850.

Mohammed R.A.A., Ellis I.O., Elsheikh S., Paish E.C., Martin S.G. (2009) Lymphatic and angiogenic characteristics in breast cancer: morphometric analysisand prognostic implications. Breast Cancer Res. Treat. 113: 261-273.

Nguyen D.X., Bos P.D., Massagué J. (2009) Metastasis: from dissemination to organ-specific colonization. Nat. Rev. Cancer 9: 274-284.

Niles A.L., Moravec R., Riss T.L. (2008) Update on in vitro cytotoxicity assays for drug development. Expert Opin. Drug Discov. 3: 655-669.

Schröterová L., Králová V., Vorácová A., Hasková P., Rudolf E., Cervinka M. (2009) Antiproliferative effects of selenium compounds in colon cancer cells: comparison of different cytotoxicity assays. Toxicol. In Vitro 23: 1406-1411.

Su J.-L., Yang P.-C., Shih J.-Y., Yang C.-Y., Wei L.-H. et al. (2006) The VEGF-C/FIt-4 axis promotes invasion and metastasis of cancer cells. Cancer Cell 9: 209-223.

Su J.-L., Chen P.-S., Chien M.-H., Chen P.B., Chen Y.-H., Lai C.-C., Hung M.-C., Kuo M.-L. (2008) Further evidence for expression and function of the VEGF-C/VEGFR-3 axis in cancer cells. Cancer Cell 13: 557-560.

Sun P., Gao J., Liu Y.L., Wei L.W., Wu L.P., Liu Z.Y. (2008) $R N A$ interference (RNAi)-mediated vascular endothelial growth factor-C (VEGF-C) reduction interferes with lym- 
phangiogenesis and enhances Epirubicin sensitivity of breast cancer cells. Mol. Cell. Biochem. 308: 161-168.

Timoshenko A.V, Rastogi S., Lala P.K. (2007) Migrationpromoting role of VEGF-C and VEGF-C binding receptors in human breast cancer cells. Br. J. Cancer 97: 1090-1098.

Ui-Tei K., Naito Y., Takahashi F., Haraguchi T., Ohki-Hamazaki H., Juni A., Ueda R., Saigo K. (2004) Guidelines for the selection of highly effective siRNA sequences for mammalian and chick RNA interference. Nucl. Acids Res. 32: 936-948. 